Article

\title{
Loss of the Mitochondrial Fission GTPase Drp1 Contributes to Neurodegeneration in a Drosophila Model of Hereditary Spastic Paraplegia
}

\author{
Philippa C. Fowler ${ }^{1}$, Dwayne J. Byrne ${ }^{1,2}$, Craig Blackstone ${ }^{2(1)}$ and Niamh C. O'Sullivan ${ }^{1, *(1)}$ \\ 1 UCD School of Biomolecular and Biomedical Sciences, UCD Conway Institute, University College Dublin, \\ Dublin 4, Ireland; philippa.fowler@ucdconnect.ie (P.C.F.); dwayne.byrne@ucdconnect.ie (D.J.B.) \\ 2 Cell Biology Section, Neurogenetics Branch, National Institute of Neurological Disorders and Stroke, \\ National Institutes of Health, Bethesda, MD 20892, USA; blackstc@ninds.nih.gov \\ * Correspondence: niamh.osullivan@ucd.ie; Tel.: +353-1-7166762
}

Received: 25 August 2020; Accepted: 15 September 2020; Published: 17 September 2020

\begin{abstract}
Mitochondrial morphology, distribution and function are maintained by the opposing forces of mitochondrial fission and fusion, the perturbation of which gives rise to several neurodegenerative disorders. The large guanosine triphosphate (GTP)ase dynamin-related protein 1 (Drp1) is a critical regulator of mitochondrial fission by mediating membrane scission, often at points of mitochondrial constriction at endoplasmic reticulum (ER)-mitochondrial contacts. Hereditary spastic paraplegia (HSP) subtype SPG61 is a rare neurodegenerative disorder caused by mutations in the ER-shaping protein Arl6IP1. We have previously reported defects in both the ER and mitochondrial networks in a Drosophila model of SPG61. In this study, we report that knockdown of Arl6IP1 lowers Drp1 protein levels, resulting in reduced ER-mitochondrial contacts and impaired mitochondrial load at the distal ends of long motor neurons. Increasing mitochondrial fission, by overexpression of wild-type Drp1 but not a dominant negative Drp1, increases ER-mitochondrial contacts, restores mitochondrial load within axons and partially rescues locomotor deficits. Arl6IP1 knockdown Drosophila also demonstrate impaired autophagic flux and an accumulation of ubiquitinated proteins, which occur independent of Drp1-mediated mitochondrial fission defects. Together, these findings provide evidence that impaired mitochondrial fission contributes to neurodegeneration in this in vivo model of HSP.
\end{abstract}

Keywords: endoplasmic reticulum; mitochondria; fission; neurodegeneration; autophagy; Drosophila

\section{Introduction}

Mitochondria are highly dynamic organelles, constantly changing their size, shape, number, and location throughout cells through the opposing forces of fission and fusion [1]. These mitochondrial processes are tightly regulated to maintain mitochondrial health, which is particularly important in cells with high energy demands such as neurons. Mitochondrial fission contributes to quality control by enabling the removal of damaged mitochondria. Defects in mitochondrial fission result in a hyperfused network consisting of highly elongated and inter-connected mitochondria [1,2]. The guanosine triphosphate (GTP)ase dynamin-related protein (Drp1) is the integral facilitator of mitochondrial fission. Drp1 is recruited to form a multimeric ring-like structure around the mitochondrial outer membrane, which undergoes GTP hydrolysis to constrict the mitochondrial membrane leading to a scission event [3,4]. Mitochondrial fission mediated by Drp1 is vital during neuronal differentiation and development $[5,6]$. Similarly, in adult neuronal axons, tight regulation of mitochondrial fission by Drp1 is essential: loss of Drp1 is found to compromise mitochondrial bioenergetics and synaptic 
function [7], while a role for Drp1 has been suggested in several neurodegenerative diseases including Alzheimer's disease [8,9], Parkinson's disease [10] and amyotrophic lateral sclerosis (ALS) [11].

Coordination of Drp1-mediated mitochondrial fission also involves the endoplasmic reticulum (ER). ER tubules form contacts marking sites for mitochondrial fission [12] while the ER-localised inverted formin 2 (IFN2) mediates the initial mitochondrial constriction via polymerisation of actin filaments [13]. Furthermore, the ER functions as a platform for Drp1 oligomerisation before transfer to mitochondria by the Drp1 receptors Mff, Fis1, MiD49 and MiD51 to contribute to fission [14]. Dynamic post-translational modification of Drp1 alters its ability to promote mitochondrial fission and through its functions, the ER additionally contributes to the regulation of these modifications. Increased cytosolic calcium promotes activation of Drp1 by dephosphorylation at S637 [15,16], while the ER resident protein disulfide isomerase (PDI) has been shown to alter S-nitrosylation and phosphorylation of Drp1 to modify mitochondrial morphology in neurons [17].

Hereditary spastic paraplegias (HSPs) are a genetically heterogeneous group of disorders characterised by degeneration of the longest motor neurons in the corticospinal tract, resulting in lower limb spasticity. The most common mutations causing HSP are loss-of-function mutations in genes encoding ER-shaping proteins which function to regulate the organisation of the ER network [18]. Given the important role of the ER in the regulation of mitochondrial fission, it has been hypothesised that dysregulation of this process may contribute to neurodegeneration in HSP. Animal models of HSP generated by loss of the ER-shaping proteins Atlastin, Reticulon or ARL6IP1 result in mitochondrial elongation and defective mitochondrial fission in neurons $[19,20]$. Fibroblasts from HSP patients with mutations in the ER-shaping protein REEP1 also display elongated mitochondria and hyperphosphorylated Drp1 compared to healthy controls which can be rescued by overexpression of Drp1 or inhibiting Drp1 S637 phosphorylation [21]. Understanding the role of Drp1-mediated mitochondrial fission in HSP-associated neurodegeneration is important as it may serve as a potential therapeutic target for some forms of these disorders.

In this study, we set out to investigate the role of mitochondrial fission in mediating neurodegeneration in a Drosophila model of HSP generated by targeted knockdown of the ER-shaping protein Arl6IP1. We show that reduction in Arl6IP1 in vivo decreases levels of Drp1, reduces ER-mitochondrial contacts and increases mitochondrial elongation in motor neuron axons. Increasing mitochondrial fission by overexpression of Drp $1^{\mathrm{WT}}$, but not dominant negative Drp $1^{\mathrm{K} 38 \mathrm{~A}}$, rescues axonal mitochondrial morphology and partially restores the progressive locomotor defects caused by knockdown of Arl6IP1. However, not all defective processes in Arl6IP1 RNAi flies are restored by overexpression of Drp1. We find evidence of disrupted protein processing in Arl6IP1 RNAi flies, specifically impaired induction of autophagy and increased aggregation of ubiquitinated proteins, which occurs independent of Drp1-mediated mitochondrial fission defects.

\section{Materials and Methods}

\subsection{Drosophila Stocks and Conditions}

Flies were raised on standard yeast, dextrose, cornmeal and agar food at $25{ }^{\circ} \mathrm{C}$ with a $12: 12$ light-dark cycle and transferred to fresh vials every 2-3 days. For Arl6IP1 knockdown experiments, the fly lines used were $w^{1118}$ GD and KK control stocks 60,000 and 60,100 and UAS-Al6IP1-RNAi GD and KK (line numbers 5894 and 10,790, respectively), all obtained from the Vienna Drosophila RNAi Centre, www.vdrc.at [22]. Both libraries were constructed by inserting inverted repeats of known genes into Drosophila, with the key difference being that GD library insertions are P-element based transgenes with random insertion sites, whereas the KK library contains phiC31-based transgenes with a single, defined insertion site. Phenotypes were analysed in RNAi knockdown lines compared to their appropriate controls. To study Drp1, the fly lines used were UAS-Drp1 provided by Mel Feany (Harvard Medical School) [23], Drp1:: FLAG-FlAsH-HA obtained from the Bloomington Stock Centre [24] and UAS-Drp $1^{w t} H A$ and UAS-Drp $1^{K 38 A} H A$ provided by Prof. Jongkyeong Chung (Korea 
Advanced Institute of Science and Technology) [25]. UAS lines were crossed to da-GAL4 [26] for the generation of samples used for sqPCR, $n S y b-G A L 4$ [27] for the generation of crosses used for behavioural studies, OK6-GAL4 [28] for mitochondrial analysis and Cg-GAL4 [29] for larval fat body analysis. Other fly stocks used were mito::GFP (stock number 8443) [30] and the autophagosomal marker GFP-mCherry-Atg8a (stock number 37,749) [31] both obtained from the Bloomington Stock Centre.

\subsection{Semi-Quantitative PCR}

Total RNA was purified from ten third instar stage larvae (L3) via treatment with TRIzol reagent (Invitrogen, Paisley, UK) as previously described [19]. Primers used were: Rp49-F: 5'-CCGACCACGTTACAAGAACTCTC-3'; Rp49-R: 5' -CGCTTCAAGGGA CAGTATCTGA-3'; Arl6IP1-F: 5'-GGTGCTGTGGTACCTGGACT-3'; Arl6IP1-R: 5'-CCAT AGCCAAAAGACCCAAA-3' ; Drp1-F: 5' -ACTGGTGCTCCAGCTGATCT-3'; Drp1-R: 5' - GATGGAGTTCGGGTTCTCAA-3'. PCR conditions were: $94{ }^{\circ} \mathrm{C}$ for $45 \mathrm{~s}, 60{ }^{\circ} \mathrm{C}$ for $45 \mathrm{~s}$ and $72{ }^{\circ} \mathrm{C}$ for $1 \mathrm{~min}$, repeated for 22 cycles for $R p 49$, 26 cycles for Arl6IP1 and 29 cycles for Drp1. Following PCR, products were visualized via a gel containing ethidium bromide.

\subsection{Immunoblot Analysis}

Soluble protein lysate was purified from 20 adult Drosophila as described previously [32]. Samples were run on $10 \%$ polyacrylamide gels and electrophoretically transferred to nitrocellulose membranes $(0.45 \mu \mathrm{m}, \mathrm{GE}$ Healthcare Sciences, Amersham, UK). Nitrocellulose membranes were then blocked in 5\% milk and incubated with primary antibodies against the HA-epitope tag (MMS-101P-100, Eurogentec; Liege, Belgium) and tubulin (T9026, Sigma; Dorset, UK). Bands were subsequently visualised using anti-mouse IgG antibodies conjugated with IR800 fluorescence dye (\#SAS-35521, Thermo Fisher Scientific; Waltham, MA, USA). Membranes were imaged using the LI-COR Odyssey Infrared Imaging System, and intensities were determined using ImageJ $1.48 \mathrm{v}$.

\subsection{Histology and Immunomicroscopy}

Immunostaining of Drosophila larvae was carried out as described previously [19]. Briefly, L3, or late L2 for analysis of autophagic flux, larvae were dissected in chilled in Ca ${ }^{+2}$-free HL3 solution [33], fixed in 4\% formaldehyde in PBS for $30 \mathrm{~min}$, permeabilised in $0.1 \%$ Triton X-100 in PBS and incubated with primary antibodies against HA (as above), Dlg (4F3, Hybridoma; Iowa City, IA, USA), GFP (A-6455, Thermo Fisher Scientific; USA), HRP (P7899, Sigma) and FK2 (BML-PW8810, Enzo Life Sciences; Farmingdale, NY, USA). Following incubation with the appropriate secondary antibodies, fixed preparations were mounted in Vectashield containing the nuclear stain DAPI (Vector Laboratories; Oxfordshire, UK) and were viewed using an Olympus IX81 confocal head mounted on an Olympus Fluoview FV-1000 microscope. All images were acquired using a 60×/1.35 NA objective and FV10-ASW cer.04.01 software. All axonal analyses were conducted by imaging motor axon bundles passing through segment A7. Similarly, analyses of neuromuscular junctions (NMJs) were conducted by imaging muscles 6 and 7 at segment A7. Z-stacks of larval fat bodies were imaged at approximately 2- $\mu \mathrm{m}$ intervals and were subsequently converted into maximum intensity projections for image analysis.

\subsection{Image Analysis}

All image analysis was carried out blind to genotype.

\subsubsection{Drp1 Localisation Analysis}

For Drp1 localisation studies, mitochondrial fission and post fission sites were quantified by measuring the number of interactions between mitochondria and Drp1::HA, where mitochondrial fission sites were defined by Drp1 staining at points of decreased mito::GFP staining and post-fission sites were defined by Drp1 staining at the ends of mito::GFP tubules. 


\subsubsection{Mitochondrial Analysis}

Mitochondrial staining at NMJs was quantified in ImageJ. Mean grey intensity values of mito::GFP within terminal synaptic boutons were normalised to bouton area as defined by the post-synaptic protein Dlg staining. For mitochondrial circularity, images were first thresholded and converted to binary images in ImageJ. The watershed function was used on binarized images to separate adjoined mitochondria. Mitochondrial circularity was quantified using the Shape Descriptors option in the ImageJ/Analyse menu which uses the equation $4 \pi \times$ [Area]/[Perimeter] 2 to generate a value between 0 and 1 , where 1 is a perfect circle and as the value approaches 0 it becomes increasingly elongated.

\subsubsection{Autophagic Flux Analysis}

Co-localisation of Atg8-mCherry and GFP puncta was quantified manually using the threshold function in ImageJ. For this, the channel corresponding to mCherry was thresholded to highlight individual puncta and converted into a binary image; the watershed function was used to separate adjoining puncta. Using this as a mask, the mean grey values of puncta were subsequently quantified in channels corresponding to mCherry and $\alpha$ GFP. Red: green (mCherry: $\alpha \mathrm{GFP}$ ) ratios were calculated by dividing the mean grey values in the red channel by the green channel, where an increase represents a drop in $\alpha \mathrm{GFP}$ signal and therefore an increased formation of autolysosomes.

\subsubsection{Quantification of Ubiquitinylated Proteins}

To quantify Fk2 staining within terminal synaptic boutons, mean grey intensity values were quantified and normalised to bouton area using HRP as a marker for neuronal membranes.

\subsection{Live Mitochondrial Trafficking in Axons}

To examine axonal mitochondrial trafficking, L3 stage larvae were dissected in HL3 supplemented with $1 \mathrm{mM} \mathrm{Ca}^{2+}$ and $4 \mathrm{mM} \mathrm{L-glut} \mathrm{pre-warmed} \mathrm{to} 25^{\circ} \mathrm{C}$. Images were acquired using a Zeiss AxioImager M1 upright fluorescent microscope with a $63 \times / 0.95$ NA objective water dipping lens every $2 \mathrm{~s}$ for $2 \mathrm{~min}$ to generate a movie. Dissected larvae were imaged no later than $30 \mathrm{~min}$ from the time of dissection. To determine average mitochondrial speed, at least 3 kymographs were generated per movie using the multi-kymograph plugin in Image J. Individual mitochondrial velocities were determined from the kymographs using the segmented line tool to trace mitochondrial distance over time, and average anterograde/retrograde speed was calculated using the read velocities from tsp macro. The proportion of mobile mitochondria was determined by manual categorisation of the movement of mitochondria within randomly placed $10 \mu \mathrm{m}^{2}$ boxes, with an average of $2-3$ boxes analysed per movie.

\subsection{Electron Microscopy}

Early L2 larvae, which could be accommodated by our instrumentation, were subjected to high pressure freezing using a BAL-TEC HPM010 freezer (ABRA Fluid AG; Widnau, Switzerland). Frozen larvae were transferred into cryovials containing a pre-cooled mix of $1 \%$ osmium tetroxide, $0.1 \%$ uranyl acetate in $95 \%$ acetone $/ 5 \% \mathrm{H}_{2} \mathrm{O}$. Specimens subsequently underwent freeze substitution for $48 \mathrm{~h}$ at $-80{ }^{\circ} \mathrm{C}$, then were transferred to $-20{ }^{\circ} \mathrm{C}$ overnight, removed and allowed to reach room temperature for $\sim 4 \mathrm{~h}$. Following a rinse in pure acetone, specimens underwent propylene oxide then propylene oxide/epoxy resin infiltration, before being embedded in 100\% resin. Ultrathin sections $(80 \mathrm{~nm})$ were obtained using a diamond knife and microtome, collected on copper grids and subsequently post-stained with uranyl acetate and lead citrate. Sections were imaged at $120 \mathrm{kV}$ using a FEI Technai 12 transmission electron microscope (EM) and at captured magnifications of $9000 \times$ and 25,000x. EM images were collected from 3-4 larvae per genotype, with a minimum of seven epidermal cells per larvae. 


\subsection{Ultrastructural Analysis}

For ER and mitochondrial analyses, the free hand tool in ImageJ was used to manually trace and measure ER and mitochondrial branch length. For ER-mitochondrial contact analyses, the circumference of each mitochondrion and the proportions of mitochondrial surface closely opposed to the ER ( $<30 \mathrm{~nm}$ ) were calculated. The number and length of individual ER-mitochondrial contacts were similarly calculated. The line tool in ImageJ was used to measure the distance between the ER and mitochondria to determine contact point thickness. All image analyses were carried out blind to genotype.

\subsection{Behavioural Analysis}

All behavioural assays were conducted on are progeny of $n S y b-G A L 4$ flies crossed to appropriate $w^{1118}$ control stocks: UAS-Arl6IP1 RNAi (Arl6IP1 RNAi), UAS-Arl6IP1 RNAi.UAS-Drp1 ${ }^{\text {WT }}$ (Arl6IP1 $\left.\mathrm{RNAi}+\operatorname{Drp} 1^{W T}\right)$ or UAS-Arl6IP1 RNAi.UAS-Drp1 ${ }^{\mathrm{K} 38 A}\left(\right.$ Arl6IP1 RNAi + Drp1 $\left.{ }^{\mathrm{K} 38 A}\right)$. Larval locomotor assays were conducted on 10 larvae per genotype per day by quantifying distance crawled in $1 \mathrm{~min}$, as previously described [34]. Assessment of adult locomotion was determined using a negative geotaxis assay. Briefly, age-matched male flies were separated into groups of 10 individuals per genotype that were tested together under the same conditions once a week over 36 days. The proportion of flies climbing to the top of a vertical glass vial $(10 \mathrm{~cm}$ length, $2.5 \mathrm{~cm}$ diameter) over $15 \mathrm{~s}$ was determined. For survival assays, male flies from climbing assays were transferred into fresh media every 2-3 days and mortality was scored daily.

\subsection{Statistical Analysis}

All data were exported to Prism 5 (GraphPad Software, Inc.; San Diego, CA, USA) for statistical analysis. For larval and adult locomotor assays and all ER and mitochondrial analyses, statistical significance was determined using one-way ANOVA and Tukey's multiple comparison post-hoc tests. Life span assays were analysed using the Kaplan Meier log-rank test. For protein expression analysis, statistical significance was determined using a two-tailed $t$-test.

\section{Results}

\subsection{Mitochondrial Fission Is Disrupted in an Arl6IP1 Knockdown Model of HSP}

Previous work in our lab has found that knockdown of the ER-shaping protein Arl6IP1 results in elongation of axonal mitochondria, suggestive of impaired mitochondrial fission [19]. To understand this effect mechanistically, we investigated whether levels of the mitochondrial fission protein Drp1 are disrupted in this model of HSP. Analysis of mRNA expression levels detected no significant change in Drp1 in Arl6IP1 RNAi Drosophila (Figure 1A). Since we were unable to source an antibody which detected Drosophila Drp1, we used a Drp1::FLAG-FlAsH-HA fusion line to quantify endogenous levels of Drp1 [24]. Analysis of the $82 \mathrm{kDa}$ band detected by HA antibodies, corresponding to full length Drp1, is significantly reduced in Arl6IP1 RNAi flies compared to controls (Figure 1B). 


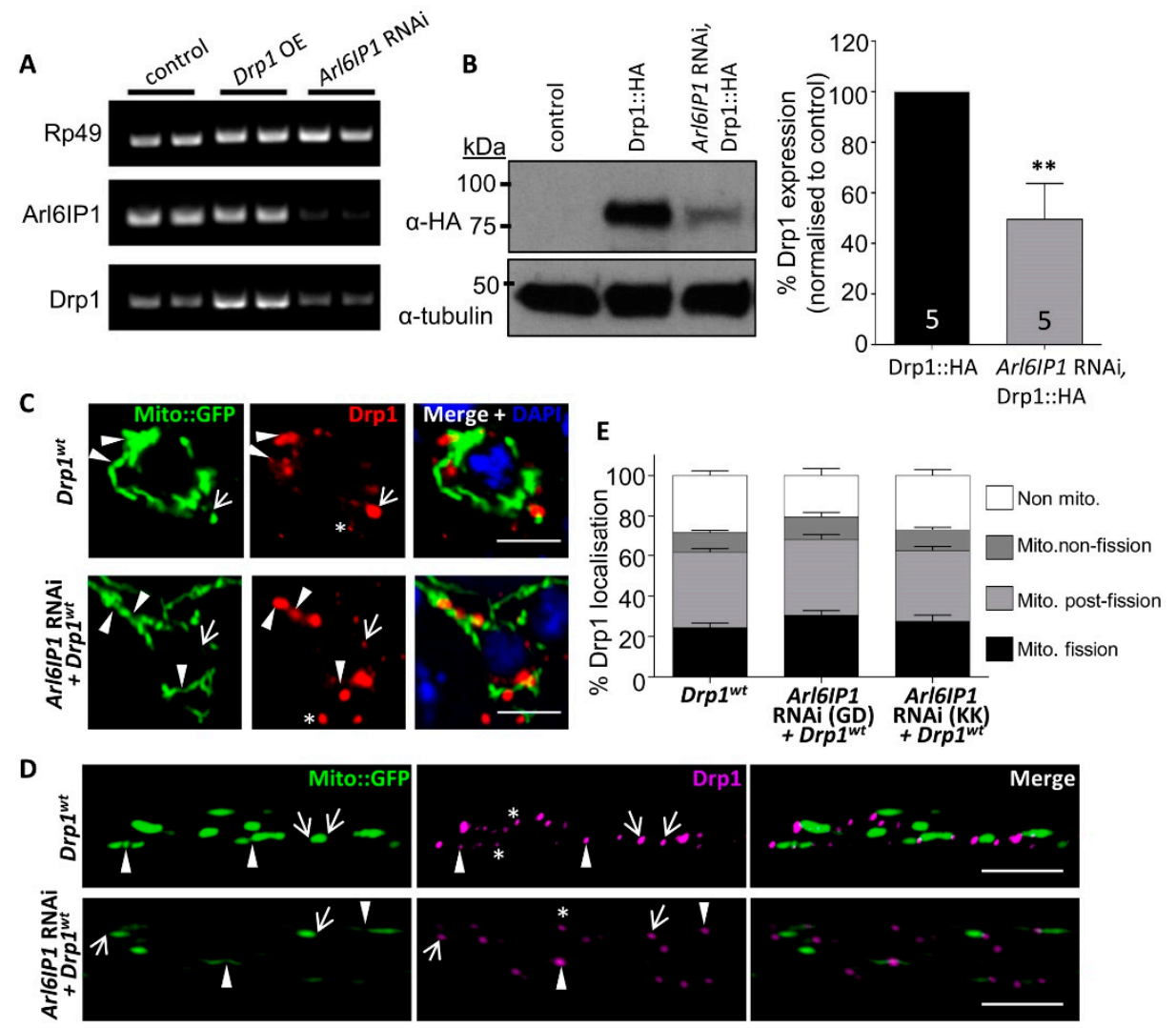

Figure 1. Decreased levels of the mitochondrial fission protein Drp1 in Arl6IP1 RNAi Drosophila. (A) Polymerase chain reaction (PCR) amplification of Rp49, Arl6IP1 and Drp1 cDNA from progeny of $w^{1118}$ (control), UAS-Drp1 (Drp1 OE) or UAS-Arl6IP1 RNAi (GD) (Arl6IP1 RNAi) crossed to da-GAL4 flies. (B) Western blot analysis of protein lysates from progeny of FLAG-FlAsH-HA Drp1 fusion (Drp1::HA) or Arl6IP1 RNAi (GD) with FLAG-FlAsH-HA Drp1 (Arl6IP1 RNAi, Drp1::HA) crossed to da-GAL4 flies. Data are expressed as means \pm SEM ( $n=5$ independent experiments), and values significantly different from control were determined by two-tailed $t$-test $(* *, p<0.01)$. (C,D) Representative confocal images of L3 larvae showing Drp1 and mitochondria in motor neuron cell bodies (C) and axons (D). Larvae are progeny of $o k 6-G A L 4$, mitoGFP crossed to UAS-Drp $1^{w t} H A$ (Drp $\left.1^{w t}\right)$ or UAS-Arl6IP1 RNAi (GD), UAS-Drp $1^{w t} H A\left(\right.$ Arl6IP1 RNAi + Drp $\left.1^{w t}\right)$ flies. Drp1 puncta co-localising with mitochondria were primarily detected at areas of reduced mitochondrial staining (arrowheads; likely during a fission event) or to the end of mitochondrial tubules (arrows; likely post-fission), while a subset do not colocalise with mitochondrial staining (asterix). (E) Quantifications of the proportion of Drp1 puncta within axon bundles localised to points of: mitochondrial constriction (Mito. Fission), ends of mitochondrial tubules (Mito. post-fission), mitochondria generally (Mito. non-fission), and independent of mitochondria (Non mito,) ( $n=6-18$ larvae). Scale bars $=5 \mu \mathrm{m}$.

To restore Drp1 expression in Arl6IP1 RNAi flies, we used a HA-tagged UAS-Drp $1^{\text {wt }}$ line to increase the expression of Drp1 [25]. Within cells, Drp1 exists as a cytoplasmic pool, with oligomers localised to mitochondria, ER and peroxisomes [14]. Immunostaining of larval motor neurons demonstrates that Drp1 punta localise primarily to mitochondria, though 20-28\% of Drp1 does not colocalise with mitochondria and likely represents ER- and peroxisomal-localised Drp1 [14]. On mitochondria, Drp1 puncta were largely localised to areas of constricted mitochondrial staining, likely reflecting an ongoing fission event, or at the ends of mitochondrial tubules, likely marking sites where fission has occurred (Figure 1C,D) [35]. This suggests that overexpressed Drp1 functions to promote mitochondrial fission within motor neurons. Importantly, overexpressed Drp1 marks fission events within Arl6IP1 
knockdown lines to a similar level, with no differences in the localisation frequencies of Drp1 puncta within axon bundles as assessed using one-way ANOVA across the three genotypes studied (Figure 1E).

The ER contacts mitochondria to mark sites for mitochondrial fission [12]. We used electron microscopy to quantify ER-mitochondrial contacts in vivo as confocal microscopy lacks the sufficient resolution to reveal details of the interactions between these organelles which involve membrane apposition of $\sim 10-30 \mathrm{~nm}$ (Figure 2) [36]. Ultrastructural analysis revealed that the branch length of ribosome-rich rough ER is unaffected in any of the genotypes studied (Figure 2C). Knockdown of Arl6IP1 leads to a striking elongation of mitochondria in epidermal cells compared to controls, as indicated by an increase in the average mitochondrial branch length (Figure 2D). To quantify ER-mitochondrial interactions, the percentage of the outer mitochondrial surface in close opposition with the ER $(<30 \mathrm{~nm})$ (Figure 2E) and the number of ER-mitochondrial contacts per mitochondrion (Supplemental Figure S1A) was calculated, revealing a significant decrease in the extent of ER-mitochondrial contacts in Arl6IP1 RNAi larvae compared to controls. We saw no change in the mean length or thickness of ER-mitochondrial contacts (Supplemental Figure S1B,C). Conversely, overexpression of Drp1 in Arl6IP1 RNAi larvae blocks mitochondrial elongation and increases the proportion of the mitochondrial membrane in contact with the ER. Together, these findings point to impaired Drp1-mediated mitochondrial fission in an Arl6IP1 knockdown model of HSP.

\subsection{Increased Drp1 Activity Rescues Mitochondrial Morphology and Neurodegeneration in Arl6IP1 Knockdown Flies}

We wanted to validate that increased mitochondrial fission driven by overexpression of Drp1 is mediating the observed rescue in mitochondrial morphology detected in Arl6IP1 RNAi Drosophila. To accomplish this, we generated transgenic flies expressing Arl6IP1 RNAi in combination with overexpression of a wild type Drp1 (Drp $\left.{ }^{\mathrm{WT}}\right)$ or a dominant negative Drp1 mutant $\left(\operatorname{Drp} 1^{\mathrm{K} 38 \mathrm{~A}}\right)$ and mitochondrial morphology and load within axons of long motor neurons was studied. Consistent with previous results, knockdown of Arl6IP1 results in elongation of axonal mitochondria and reduced mitochondrial load in terminal boutons within posterior neuromuscular junctions (NMJs) [19] (Figure 3A,B). The loss of mitochondrial load from the terminal boutons is not the result of defective mitochondrial trafficking, since no change in mitochondrial flux or trafficking speed was detected in Arl6IP1 RNAi flies compared to controls (Figure 3C). Instead, we suggest that the reduction in mitochondrial load is due to impaired mitochondrial fission which could limit the production of daughter mitochondria at the synapse. Overexpression of Drp $1^{\mathrm{WT}}$ largely rescued axonal mitochondrial morphology and NMJ load (Figure 3A,B). However, overexpression of Drp1 ${ }^{\mathrm{K} 38 \mathrm{~A}}$ failed to rescue, and sometimes enhanced, these mitochondrial phenotypes (Figure $3 \mathrm{~A}, \mathrm{~B}$ ). This demonstrates that altered mitochondrial morphology in Arl6IP1 RNAi motor neuron axons can be rescued by increasing mitochondrial fission.

Neuronal knockdown of Arl6IP1 in Drosophila results in progressive locomotor deficits recapitulating a key characteristic of HSP [19]. We now show that both larval and adult locomotor defects in Arl6IP1 RNAi Drosophila are largely restored by overexpression of Drp1 ${ }^{\text {WT }}$ (Figure 4A,B; Tables S1 and S2). In contrast, overexpression of Drp1 ${ }^{\mathrm{K} 38 \mathrm{~A}}$ fails to rescue locomotion in Arl6IP1 RNAi larvae (Figure 4A) and significantly exacerbates the progressive locomotor defect in Arl6IP1 RNAi flies, particularly beyond 18 days old (Figure 4B; Tables S1 and S2). Survival is not consistently altered across the genotypes studied (Figure 4C; Tables S3 and S4). Together, these data show that increased mitochondrial fission can help to prevent neurodegeneration in this model of HSP. 

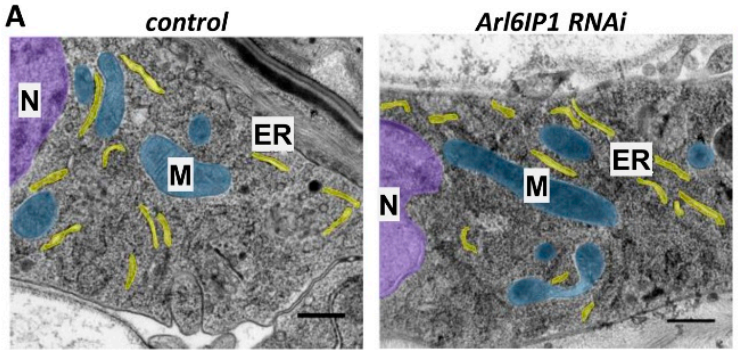

ArI6IP1 RNAi +DrP1 OE
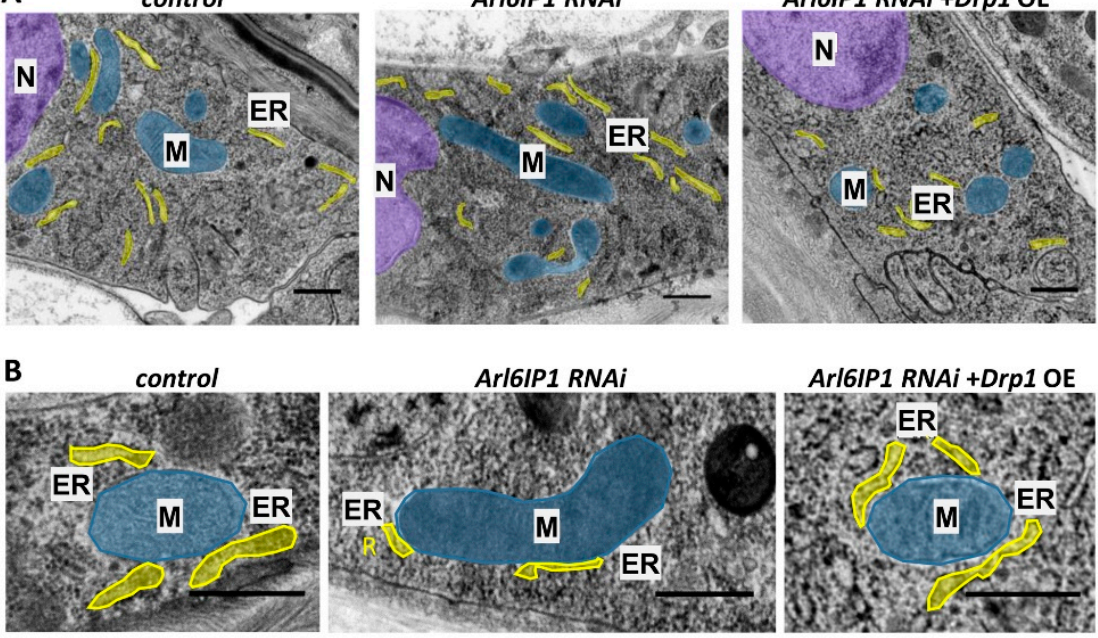

C

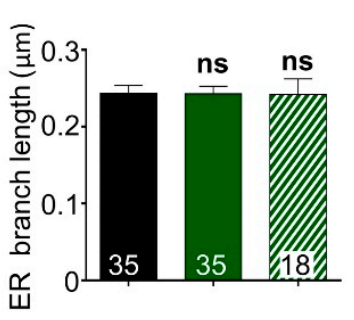

ArI6IP1 RNAi

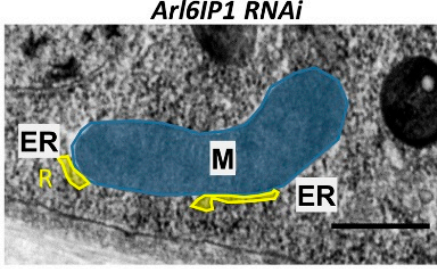

ArI6IP1 RNAi +Drp1 OE

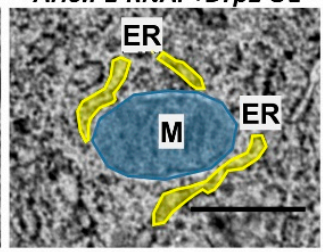

D

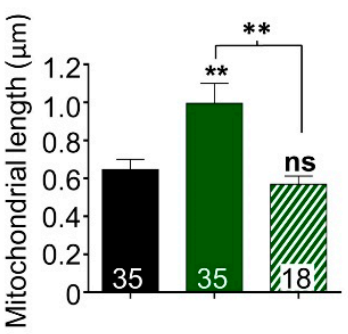

E

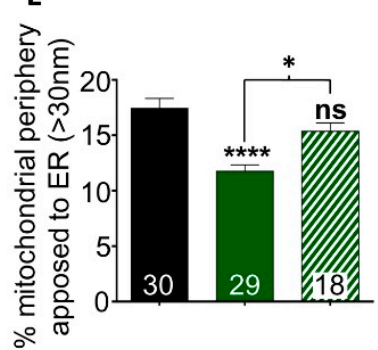

control

ArIGIP1 RNAi

TIA ArIGIP1 RNAi +Drp1 OE

Figure 2. Overexpression of Drp1 increases ER-mitochondrial contacts and blocks mitochondrial elongation in Arl6IP1 knockdown Drosophila. (A) Representative electron micrographs revealing mitochondrial (blue; M) and ER (yellow; ER) structures within larval epidermal cells. These cells were chosen as they allowed reproducible visualisation of cytoplasmic components surrounding the nucleus (purple; N). Larvae are progeny of $w^{1118}$ (control), UAS-Arl6IP1 RNAi (GD) or UAS-Arl6IP1 RNAi (GD). UAS-Drp1 (Arl6IP1 RNAi + Drp1 OE) flies crossed to da-GAL4. (B) Representative electron micrographs of ER-mitochondrial contacts in the three genotypes studied. Quantification of ER branch length (C), mitochondrial length (D) and proportion of the mitochondrial membrane contacting ER (E) in the three genotypes studied. Data are expressed as means \pm SEM ( $n=18-35$ cells from three independent experiments) and values significantly different from control were determined by one-way ANOVA and Tukey's multiple comparisons test $\left({ }^{*}, p<0.05 ;{ }^{* *}, p<0.01 ;{ }^{* * *}, p<0.0001 ; \mathrm{ns}, p>0.05\right)$. All scale bars $=5 \mathrm{~nm}$. 

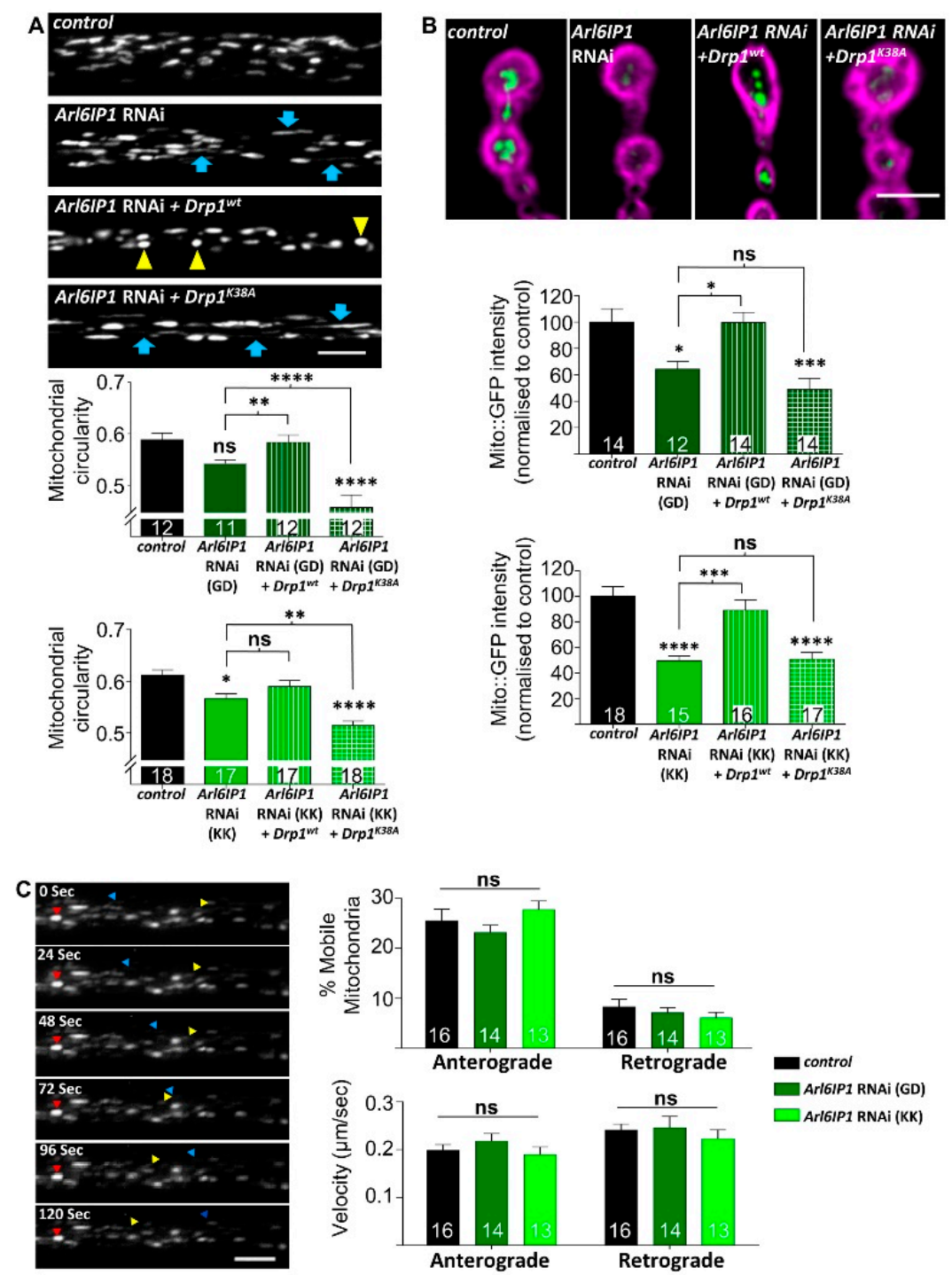

Figure 3. Increased mitochondrial fission via overexpression of $D r p 1^{w t}$ restores mitochondrial morphology and localisation within distal portions of Arl6IP1 RNAi long motor neurons. Larvae are progeny of $o k 6-G A L 4$ flies carrying mito::GFP crossed to $w^{1118}$ (control), UAS-Arl6IP1 RNAi (Arl6IP1 RNAi), UAS-Arl6IP1 RNAi.UAS-Drp1 ${ }^{W T}$ (Arl6IP1 RNAi $\left.+D r p 1^{W T}\right)$ or UAS-Arl6IP1 RNAi.UAS-Drp1 ${ }^{\text {K38A }}$ (Arl6IP1 RNAi + Drp1 ${ }^{\text {K38A }}$ ). $w^{1118}$ and UAS-Al6IP1-RNAi lines from two independent RNAi stocks (GD and KK) were tested separately. (A) Representative confocal images showing motor neuron mitochondria in posterior axon bundles. Overexpression of Drp ${ }^{\text {WT }}$ in Arl6IP1 RNAi larvae reduced mitochondrial elongation (yellow arrowheads) while overexpression of $\operatorname{Drp} 1^{\mathrm{K} 38 \mathrm{~A}}$ further increases mitochondrial elongation (blue arrows) compared to Arl6IP1 RNAi alone. Graphs show mitochondrial circularity. (B) Representative confocal images showing mitochondria (green) within terminal synaptic boutons (DLG, magenta) of posterior NMJs. Graphs show mito::GFP intensity within posterior boutons normalised to controls. Live axons expressing mito::GFP were imaged at 2 -s intervals for $2 \mathrm{~min}$ with representative stills taken every $24 \mathrm{~s}$ over the 2 -min video shown in (C). Mitochondria were categorised as stationary (red arrowhead), oscillatory or moving (anterograde-blue arrowhead, retrograde-yellow arrowhead). Graphs show proportion of moving mitochondria and mitochondrial velocity within Arl6IP1 RNAi motor neuron axons in both anterograde and retrograde direction. All data are expressed as means $\pm \operatorname{SEM}(n=11-18$ larvae from three independent experiments) and values significantly different from controls were determined by one-way ANOVA and Tukey's multiple comparisons test $\left({ }^{*}, p<0.05 ; * *, p<0.01 ; * * *, p<0.001 * * * *, p<0.0001 ; \mathrm{ns}, p>0.05\right)$. Scale bars $=5 \mu \mathrm{m}$. 
A

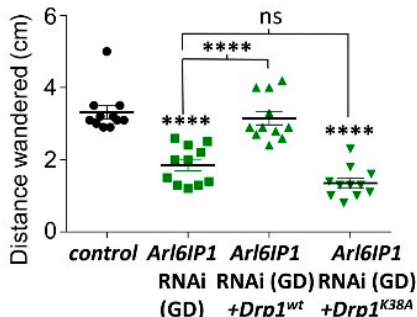

B
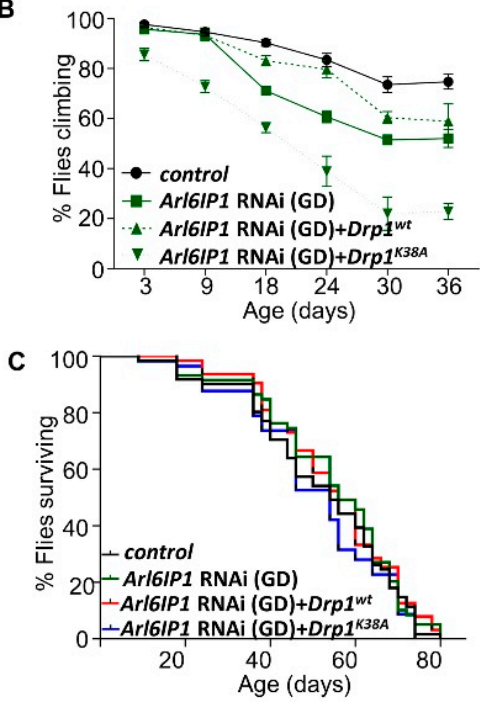
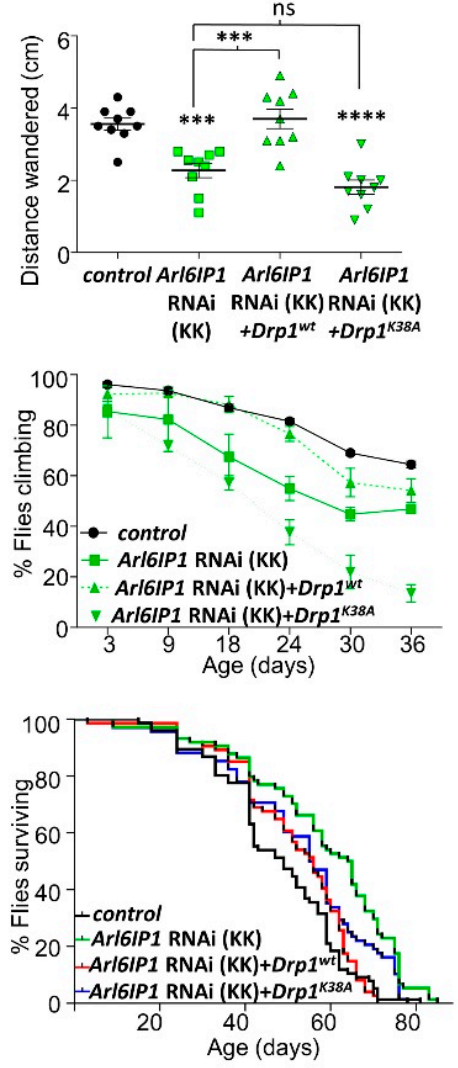

Figure 4. Increased mitochondrial fission induced by overexpression of $D r p 1^{\text {twt }}$ partially rescues locomotor deficits in Arl6IP1 RNAi Drosophila. All Drosophila are progeny of $n S y b$-GAL4 flies crossed to $w^{1118}$ (control), UAS-Arl6IP1 RNAi (Arl6IP1 RNAi), UAS-Arl6IP1 RNAi.UAS-Drp1 ${ }^{\text {WT }}$ (Arl6IP1 RNAi + $\operatorname{Drp} 1^{\text {WT }}$ ) or UAS-Arl6IP1 RNAi.UAS-Drp1 ${ }^{\text {K38A }}$ (Arl6IP1 RNAi + Drp1 $\left.{ }^{K 38 A}\right) . w^{1118}$ and UAS-Al6IP1-RNAi lines from two independent RNAi stocks (GD and KK) were tested separately. (A-C) Graphs show distance crawled by larvae in $1 \mathrm{~min}(\mathbf{A} ; n=9-10$ experiments; 10 larvae per experiment; and values significantly different from controls were determined by one-way ANOVA and Tukey's multiple comparisons test $\left(* * *, p<0.001^{* * * *}, p<0.0001 ; \mathrm{ns}, p>0.05\right)$ ), percent flies climbing in a negative geotaxis assay (B; $n=11-27$ experiments per genotypes; see Supplemental Tables S1 and S2 for statistical analysis) and lifespan assays (C; $n=57-76$ flies per genotype; see Supplemental Materials, Tables S3 and $\mathrm{S} 4$ for statistical analysis).

\subsection{Protein Processing Pathways Are Impaired in Arl6IP1 Knockdown Flies Independent of Drp1 Expression}

ER-shaping proteins have a highly conserved role in regulating autophagic degradation of the ER, from plants [37] to mammalian cells [38]. Drp1 has also been found in several studies to regulate autophagy, with reduced Drp1 leading to an accumulation of autophagosomes [39], while Drp1 overexpression enhances autophagy [6]. To investigate autophagic degradation in our model of HSP, we used the dual tagged GFP-mCherry-Atg8a marker which labels autophagosomes as yellow (with both GFP and mCherry), while low $\mathrm{pH}$ autolysosomes are labelled mostly red due to protonation quenching of GFP [40]. An increase in the red-green ratio of Atg8a puncta therefore acts as a readout of autophagic flux. During insect metamorphosis, several tissues including the fat body undergo precisely timed periods of programmed autophagy. This developmental autophagy obscures detection of starvation-induced autophagy and as a result L2 stage larvae are instead utilised to analyse induced autophagy [41]. Under fed conditions, basal autophagy in late L2 stage larvae is low and Atg8a puncta stain mostly yellow (Figure 5A). Starvation induces autophagy and within control larvae we see an expected marked increase in red Atg8a puncta, indicating the degradative completion of autophagy via the formation of autolysosomes (Figure 5A). However, we find that knockdown of Ar6IP1 impairs 
this induction of autophagy, with the red-green ratio of Atg8a puncta remaining similar in fed and starved larvae (Figure 5B). Overexpression of Drp1 fails to restore starvation-induced autophagy in Arl6IP1 RNAi larvae, indicating that this impairment occurs independent of Drp1-mediated mitochondrial fission.

A
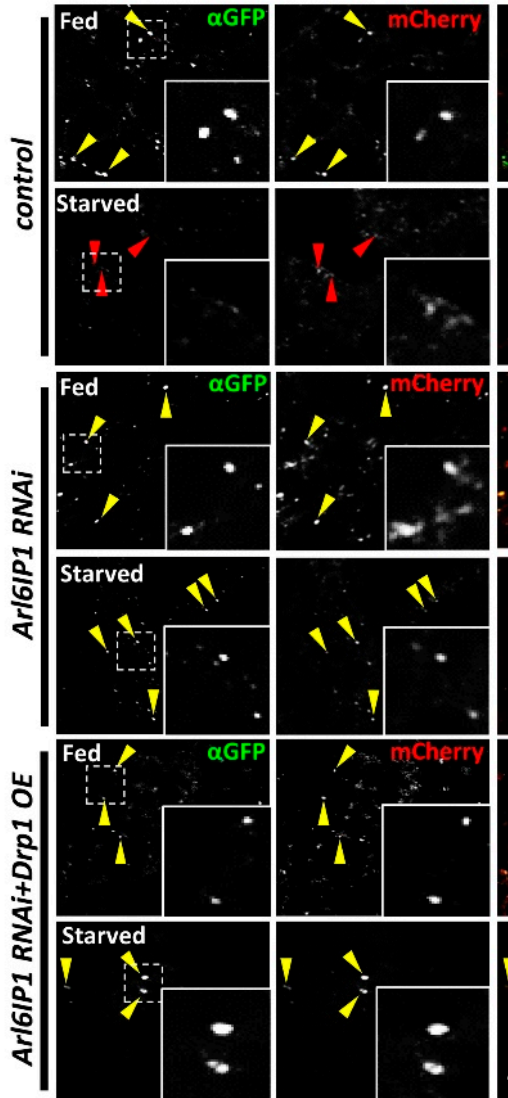
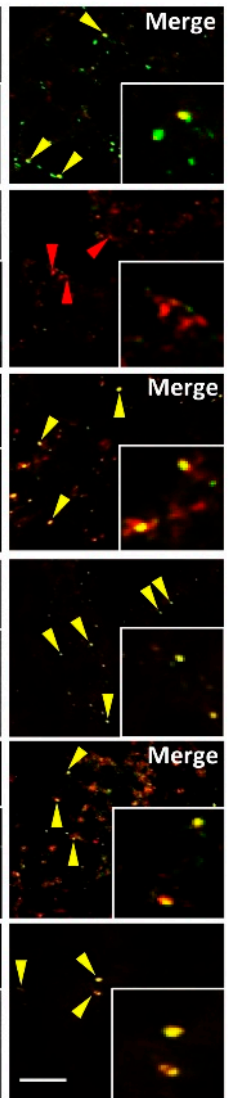

B
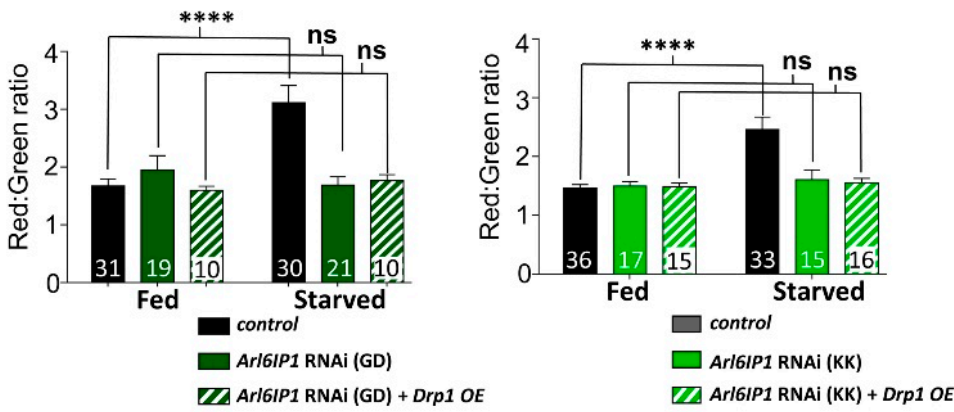

Figure 5. Knockdown of Arl6IP1 RNAi in impaired included autophagy which is not affected by overexpression of Drp1. (A) Representative confocal sections showing Drosophila fat body cells expressing the autophagosomal maker mCherry-GFP-Atg8a from late L2 larvae under fed or starved conditions. Larvae are progeny of Cg-GAL4. UAS-GFP-mCherry-Atg8a flies crossed to $w^{118}$ (controls), UAS-Arl6IP1 RNAi (Arl6IP1 RNAi) or UAS-Arl6IP1 RNAi.UAS-Drp1 (Arl6IP1 RNAi + Drp1 OE). At cytosolic $\mathrm{pH}$ both the GFP and mCherry tags are detectible (yellow arrowheads) while within the low $\mathrm{pH}$ of an autolysosome the GFP is quenched and only mCherry is detectible (red arrowheads). Scale bar $=10 \mu \mathrm{m}$. (B) Quantification of mean red (mCherry) and green (GFP) intensities of Atg8a-tagged puncta under fed and starved condition in UAS-Al6IP1-RNAi lines from two independent RNAi stocks (GD and KK) which were tested separately. Data are expressed as means \pm SEM ( $n=10-36$ larvae) and values significantly different from control were determined by one-way ANOVA and Tukey's multiple comparisons test $(* * * *, p<0.0001 ; \mathrm{ns}, p>0.05)$. 
Impaired autophagy has been reported in several subtypes of HSP [42-44] and individuals with mutations in the ER-shaping protein Atlastin 3 (associated with the related disorder hereditary sensory and autonomic neuropathy (HSAN)) have compromised autophagy [45]. However, this is the first-time disrupted autophagy has been reported in an Arl6IP1 model of HSP. To validate this finding, we looked for evidence of impaired protein clearance in our models, as attenuated autophagy has been linked to the accumulation of polyubiquitin aggregates [46,47]. Dissected larval preps were stained with Fk2 antibodies which recognise mono-and poly-ubiquitinylated substrates and free ubiquitin chains (Figure 6A). We found that knockdown of Arl6IP1 results in increased ubiquitin-positive staining within synaptic boutons of distal NMJs of Arl6IP1 RNAi flies which is not rescued by overexpression of Drp1 (Figure 6B). This confirms that protein degradation is impaired in Arl6IP1 RNAi flies, independent of Drp1-mediated mitochondrial fission.
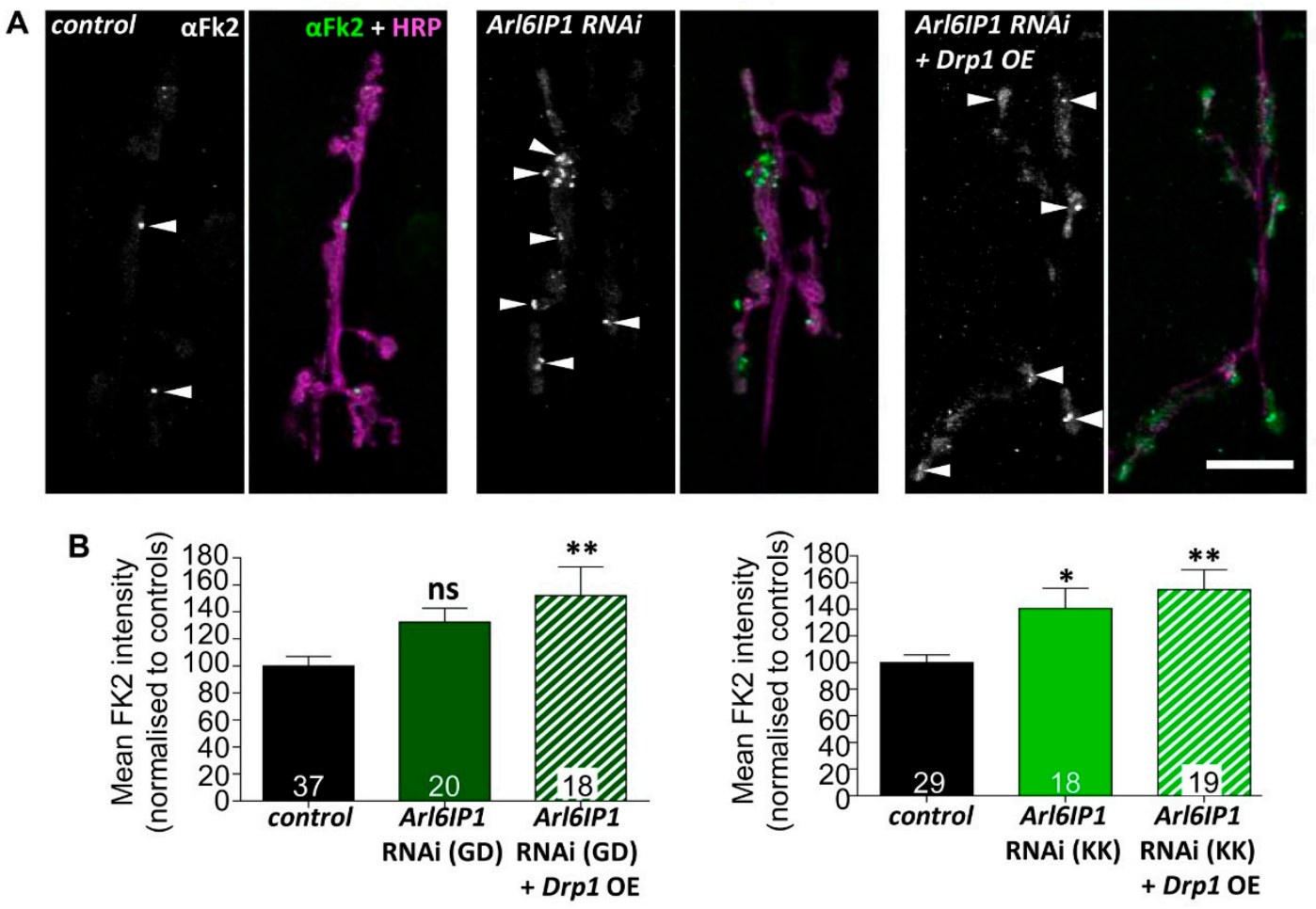

Figure 6. Knockdown of Arl6IP1 causes increased aggregation of ubiquitinated proteins in neurons which is not rescued by Drp1 overexpression. (A) Representative single confocal sections showing Fk2-positive puncta accumulation (green; white arrowheads) within posterior axons (HRP; magenta). Larvae are progeny of $d a-G A L 4$ flies crossed to $w^{1118}$ (controls), UAS-Arl6IP1 RNAi (Arl6IP1 RNAi) or UAS-Arl6IP1 RNAi.UAS-Drp1 (Arl6IP1 RNAi + Drp1 OE). Scale bar $=20 \mu \mathrm{m}$. (B) Quantification reveals increased Fk2-positive staining in NMJ boutons of flies expressing Arl6IP1 RNAi from two independent RNAi stocks (GD and KK, tested separately) compared to relevant controls. Data are expressed as means \pm SEM ( $n=18-37$ larvae from three independent experiments) and values significantly different from control were determined by one-way ANOVA and Tukey's multiple comparisons test ${ }^{*}, p<0.05$; **, $p<0.01 ; \mathrm{ns}, p>0.05)$.

\section{Discussion}

The large GTPase Drp1 is a critical regulator of mitochondrial fission, and dysregulation of the mitochondrial fission/fusion balance which has been implicated in the pathogenesis of several neurodegenerative diseases. Here, we study a Drosophila model of HSP generated by targeted knockdown of the ER-shaping protein Arl6IP1, mutations in which cause the HSP subtype SPG61 [48]. We report that Drp1 expression is decreased in this HSP model, causing reduced ER-mitochondrial contacts and impaired mitochondrial fission. Overexpression of Drp1 restores mitochondrial fission 
and partially rescues locomotor deficits, providing evidence that impaired mitochondrial fission contributes to neurodegeneration in this model of HSP.

Drp1 is a highly dynamic protein which is modulated by a vast array of physiological responses including but not limited to: exercise [49], inflammatory signalling [50] and neuronal differentiation [6]. Dysregulation of Drp1 expression has been reported in samples from patients with Alzheimer's disease [8] and ALS [11]. Furthermore, Drp1 dysregulation is detected in several models of neurodegeneration, with mutations in the AFG3-like matrix AAA peptidase subunit 2 (AFG3L2) gene, a paralog of the HSP-causing gene SPG7, and knockdown of the ER-shaping protein REEP1 both causing a reduction in the expression of Drp1 protein [21,51]. The mechanism by which Drp1 expression is altered in these models is not known, though REEP1 has been suggested to affect the stability of Drp1 by modulating its phosphorylation status [21]. We now show that Drp1 expression is decreased in an Arl6IP1 RNAi Drosophila model of HSP. This finding emphasises the importance of investigating Drp1-mediated mitochondrial fission in HSP.

There are several mechanisms by which a decrease in Drp1 levels and impaired mitochondrial fission might contribute to neurodegeneration in our model of HSP. One mechanism which must be considered is a disruption in mitophagy, the turnover and maintenance of healthy mitochondria by autophagy. Mitophagy can be regulated by Drp1 activity, with decreased Drp1-mediated mitochondrial fission resulting in hyperfused mitochondria which escape induced mitophagy [52,53]. While this can preserve energy production within a cell in the short term, long-term inhibition of mitophagy results in an accumulation of defective mitochondria contributing to neurodegeneration [54]. Several studies have reported a conserved age-related decline in mitophagy [55-57] which would be exacerbated by disorders which cause a reduction in Drp1. Here, we report that Arl6IP1 knockdown flies have impaired induced autophagy which is not restored by overexpression of Drp1. Future work to investigate basal and induced mitophagy in this model of HSP and whether these are impacted by overexpression of Drp1 would help to determine whether this might be contributing to neurodegeneration.

Drp1-mediated mitochondrial fission also regulates how post-mitotic neurons respond to oxidative stress. Reduced Drp1 results in an increase in reactive oxygen species (ROS) and oxidative damage in mouse cerebellar neurons, while treatment with antioxidants reduces mitochondrial elongation and neuronal death [58]. Drp1 may also impact apoptosis in affected neurons however either increased or decreased Drp1 activity has been shown to induce apoptosis in differentiating and cultured neurons $[6,59]$. Therefore, it remains unclear whether or how this might be contributing to neurodegeneration in different models.

Finally, lipid biogenesis can be disrupted by alterations to mitochondrial fission/fusion. Within the nervous system, lipid droplets (LDs) play vital roles in energy storage, glia-neuron communication and ROS management and lipid homeostasis is necessary for maintaining neuronal function and synaptic plasticity [60]. Defective mitochondrial fusion causes an accumulation of neutral lipids within lipid droplets in both mitofusin 1 knockout cells and fibroblasts from Charcot-Marie-Tooth disease (CMT) type 2A patients [61,62]. By contrast, loss of the mitofusion 1 ortholog in Drosophila, Marf, results in a marked decrease in LDs in an endocrine organ, the ring gland [63]. Most recently, Drp1 was shown to directly regulate LD formation, with targeted knockout of Drp1 resulting in an increase in large LDs within mouse adipose tissue [37]. This LD defect can be rescued by overexpression of Drp1. Disrupted ER, on which LDs form, and decreased Drp1 levels may therefore synergistically contribute to neurodegeneration in our model of HSP by disrupting LD formation. Interestingly, defects in lipid metabolism and storage within LDs are increasingly being associated with HSPs suggesting that lipid biogenesis may contribute to the pathophysiology of these diseases [64].

Given that we have found that restoring Drp1-mediated mitochondrial fission partially rescues locomotor defects in our model of HSP, it may lead us to consider whether Drp1 could be a potential therapeutic target for HSP or other neurodegenerative disorders. Several studies support the suggestion that increased Drp1-mediated mitochondrial fission can have beneficial effects in Drosophila models of Alzheimer's disease, HSP and Parkinson's disease [21,65]. In fact, even brief overexpression of Drp1 
during midlife in Drosophila reduces age-associated mitochondrial elongation, facilitates mitophagy and lowers mitochondrial reactive oxygen species (ROS) levels [57]. Moreover, these changes are accompanied by a significant increase in mobility and lifespan, further suggesting that treatments which promote Drp1-mediated mitochondrial fission could be useful to combat degenerative phenotypes. On the other hand, many other studies have emphasised that overexpression of Drp1 in vivo can have harmful effects, significantly disrupting the mitochondrial network and stress pathway induction [66]. Furthermore, inhibition of Drp1 has been shown to be neuroprotective in mammalian cellular and in vivo models of HSP and Parkinson's disease [59,67]. It is therefore likely that long-term inhibition or activation of mitochondrial fission would not be a suitable therapeutic approach due to potential disruption to mitophagy, apoptosis, or lipid biosynthesis. Instead, an approach whereby a healthy balance between mitochondrial fission and fusion is restored would be preferable.

Supplementary Materials: The following are available online at http://www.mdpi.com/2076-3425/10/9/646/s1, Figure S1: Analysis of ER-mitochondrial contacts in Arl6IP1 knockdown Drosophila. Further analysis of electro micrographs of larvae as described in Figure 2. Graphs show quantification of number of ER contacts per mitochondrion (A), total length of ER-mitochondrial contacts per mitochondrion (B) and frequency distribution of the thickness of ER-mitochondrial contacts (E) in the three genotypes studied. Data are expressed as means \pm SEM ( $n=18-30$ cells from three independent experiments) and values significantly different from control were determined by one-way ANOVA and Tukey's multiple comparisons test $\left.{ }^{* * * *}, p<0.0001 ; \mathrm{ns}, p>0.05\right)$, Table S1: Statistical comparison of climbing assays of male flies generated by crossing $n S y b$-GAL4 flies to $w^{1118}$ (control), UAS-Arl6IP1 RNAi (Arl6IP1 RNAi), UAS-Arl6IP1 RNAi.UAS-Drp1 ${ }^{\text {WT }}$ (Arl6IP1 RNAi + Drp1 ${ }^{\text {WT }}$ ) or UAS-Arl6IP1 RNAi.UAS-Drp1 ${ }^{K 38 A}$ (Arl6IP1 RNAi + Drp1 ${ }^{K 38 A}$ ), Table S2: Statistical comparison of climbing assays of male flies generated by crossing $n S y b-G A L 4$ flies to $w^{1118}$ (control), UAS-Arl6IP1 RNAi (Arl6IP1 RNAi), UAS-Arl6IP1 RNAi.UAS-Drp1 ${ }^{W T}$ (Arl6IP1 RNAi + Drp1 $\left.{ }^{W T}\right)$ or UAS-Arl6IP1 RNAi.UAS-Drp1 ${ }^{\text {K38A }}$ (Arl6IP1 RNAi + Drp1 ${ }^{\text {K38A }}$ ), Table S3: $n$ numbers and median lifespan for survival assays of male flies generated as in Tables S1 and S2, Table S4: Statistical comparison of survival assays of male flies generated as in Tables S1 and S2.

Author Contributions: P.C.F. performed the analysis of Drp1 expression and localisation, EM, mitochondrial morphology, behaviour, autophagic flux and ubiquitin accumulation. D.J.B. performed mitochondrial trafficking assays. P.C.F., D.J.B. and N.C.O. wrote and edited the manuscript and prepared the figures. C.B. commented on and reviewed the manuscript prior to submission. All authors contributed to manuscript revision. All authors have read and agreed to the published version of the manuscript.

Funding: This work was supported by the SFI-HRB-Wellcome Trust Biomedical Research Partnership. N.C.O. and P.C.F. were supported by Seed Award in Science (Grant Number 202020/Z/16/Z) awarded to N.C.O., D.J.B. is supported by a Wellcome Trust-National Institute of Health PhD studentship (Grant Number 200927/Z/16/A). C.B. is supported by the Intramural Research Program of the NINDS, NIH.

Acknowledgments: We thank J. Simpson of UCD School of Biology and Environmental Science for assistance with confocal microscopic imaging and T. O'Neill, J. Kennedy and D. Scholz of the UCD Conway Institute Imaging Core for assistance with electron microscopy analysis. Stocks obtained from the Bloomington Drosophila Stock Center (NIH P40OD018537) and the Vienna Drosophila Resource Center (VDRC, www.vdrc.at) were used in this study.

Conflicts of Interest: The authors declare no conflict of interest.

\section{References}

1. Youle, R.J.; van der Bliek, A.M. Mitochondrial fission, fusion, and stress. Science 2012, 337, $1062-1065$. [CrossRef] [PubMed]

2. Tilokani, L.; Nagashima, S.; Paupe, V.; Prudent, J. Mitochondrial dynamics: Overview of molecular mechanisms. Essays Biochem. 2018, 62, 341-360. [CrossRef] [PubMed]

3. Smirnova, E.; Griparic, L.; Shurland, D.-L.; van der Bliek, A.M. Dynamin-related protein Drp1 is required for mitochondrial division in mammalian cells. Mol. Biol. Cell 2001, 12, 2245-2256. [CrossRef] [PubMed]

4. Pernas, L.; Scorrano, L. Mito-Morphosis: Mitochondrial fusion, fission, and cristae remodeling as key mediators of cellular function. Annu. Rev. Physiol. 2016, 78, 505-531. [CrossRef]

5. Itoh, K.; Murata, D.; Kato, T.; Yamada, T.; Araki, Y.; Saito, A.; Adachi, Y.; Igarashi, A.; Li, S.; Pletnikov, M.; et al. Brain-specific Drp1 regulates postsynaptic endocytosis and dendrite formation independently of mitochondrial division. Elife 2019, 8, 11809-11822. [CrossRef] 
6. Vantaggiato, C.; Castelli, M.; Giovarelli, M.; Orso, G.; Bassi, M.T.; Clementi, E.; De Palma, C. The fine tuning of drp1-dependent mitochondrial remodeling and autophagy controls neuronal differentiation. Front. Cell. Neurosci. 2019, 13, 120. [CrossRef]

7. Shields, L.Y.; Kim, H.; Zhu, L.; Haddad, D.; Berthet, A.; Pathak, D.; Lam, M.; Ponnusamy, R.; Diaz-Ramirez, L.G.; Gill, T.M.; et al. Dynamin-related protein 1 is required for normal mitochondrial bioenergetic and synaptic function in CA1 hippocampal neurons. Cell Death Dis. 2015, 6, e1725. [CrossRef]

8. Manczak, M.; Calkins, M.J.; Reddy, P.H. Impaired mitochondrial dynamics and abnormal interaction of amyloid beta with mitochondrial protein Drp1 in neurons from patients with Alzheimer's disease: Implications for neuronal damage. Hum. Mol. Genet. 2011, 20, 2495-2509. [CrossRef]

9. Wang, W.; Yin, J.; Ma, X.; Zhao, F.; Siedlak, S.L.; Wang, Z.; Torres, S.; Fujioka, H.; Xu, Y.; Perry, G.; et al. Inhibition of mitochondrial fragmentation protects against Alzheimer's disease in rodent model. Hum. Mol. Genet. 2017, 26, 4118-4131. [CrossRef]

10. Filichia, E.; Hoffer, B.; Qi, X.; Luo, Y. Inhibition of Drp1 mitochondrial translocation provides neural protection in dopaminergic system in a Parkinson's disease model induced by MPTP. Sci. Rep. 2016, 6, 32656. [CrossRef]

11. Joshi, A.U.; Saw, N.L.; Vogel, H.; Cunnigham, A.D.; Shamloo, M.; Mochly-Rosen, D. Inhibition of Drp1/Fis1 interaction slows progression of amyotrophic lateral sclerosis. EMBO Mol. Med. 2018, 10, e8166. [CrossRef] [PubMed]

12. Friedman, J.R.; Lackner, L.L.; West, M.; DiBenedetto, J.R.; Nunnari, J.; Voeltz, G.K. ER tubules mark sites of mitochondrial division. Science 2011, 334, 358-362. [CrossRef] [PubMed]

13. Korobova, F.; Ramabhadran, V.; Higgs, H.N. An actin-dependent step in mitochondrial fission mediated by the ER-associated Formin INF2. Science 2013, 339, 464-467. [CrossRef] [PubMed]

14. Ji, W.K.; Chakrabarti, R.; Fan, X.; Schoenfeld, L.; Strack, S.; Higgs, H.N. Receptor-mediated Drp1 oligomerization on endoplasmic reticulum. J. Cell Biol. 2017, 216, 4123-4139. [CrossRef] [PubMed]

15. Cribbs, J.T.; Strack, S. Reversible phosphorylation of Drp1 by cyclic AMP-dependent protein kinase and calcineurin regulates mitochondrial fission and cell death. EMBO Rep. 2007, 8, 939-944. [CrossRef]

16. Chang, C.R.; Blackstone, C. Cyclic AMP-dependent protein kinase phosphorylation of Drp1 regulates its GTPase activity and mitochondrial morphology. J. Biol. Chem. 2007, 283, 21583-21587. [CrossRef]

17. Lee, D.; Kim, J.E. PDI-mediated S-nitrosylation of DRP1 facilitates DRP1-S616 phosphorylation and mitochondrial fission in CA1 neurons. Cell Death Dis. 2018, 9, 869. [CrossRef]

18. Blackstone, C. Converging cellular themes for the hereditary spastic paraplegias. Curr. Opin. Neurobiol. 2018, 51, 139-146. [CrossRef]

19. Fowler, P.C.; O'Sullivan, N.C. ER-shaping proteins are required for ER and mitochondrial network organization in motor neurons. Hum. Mol. Genet. 2016, 25, 2827-2837. [CrossRef]

20. Liu, X.; Guo, X.; Niu, L.; Li, X.; Sun, F.; Hu, J.; Wang, X.; Shen, K. Atlastin-1 regulates morphology and function of endoplasmic reticulum in dendrites. Nat. Commun. 2019, 10, 568. [CrossRef]

21. Lavie, J.; Serrat, R.; Bellance, N.; Courtand, G.; Dupuy, J.W.; Tesson, C.; Coupry, I.; Brice, A.; Lacombe, D.; Durr, A.; et al. Mitochondrial morphology and cellular distribution are altered in SPG31 patients and are linked to DRP1 hyperphosphorylation. Hum. Mol. Genet. 2017, 26, 674-685. [CrossRef] [PubMed]

22. Dietzl, G.; Chen, D.; Schnorrer, F.; Su, K.-C.; Barinova, Y.; Fellner, M.; Gasser, B.; Kinsey, K.; Oppel, S.; Scheiblauer, S.; et al. A genome-wide transgenic RNAi library for conditional gene inactivation in Drosophila. Nature 2007, 448, 151-156. [CrossRef] [PubMed]

23. DuBoff, B.; Götz, J.; Feany, M.B. Tau promotes neurodegeneration via DRP1 mislocalization in vivo. Neuron 2012, 75, 618-632. [CrossRef] [PubMed]

24. Verstreken, P.; Ly, C.V.; Venken, K.J.T.; Koh, T.-W.; Zhou, Y.; Bellen, H.J. Synaptic mitochondria are critical for mobilization of reserve pool vesicles at Drosophila neuromuscular junctions. Neuron 2005, 47, 365-378. [CrossRef]

25. Park, J.; Lee, G.; Chung, J. The PINK1-Parkin pathway is involved in the regulation of mitochondrial remodeling process. Biochem. Biophys. Res. Commun. 2009, 378, 518-523. [CrossRef]

26. Perrin, L.; Bloyer, S.; Ferraz, C.; Agrawal, N.; Sinha, P.; Dura, J.M. The Leucine Zipper Motif of the Drosophila AF10 Homologue Can inhibit PRE-mediated repression: Implications for leukemogenic activity of human MLL-AF10 fusions. Mol. Cell. Biol. 2003, 23, 119-130. [CrossRef]

27. Bushey, D.; Tononi, G.; Cirelli, C. The Drosophila fragile X mental retardation gene regulates sleep need. J. Neurosci. 2009, 29, 1948-1961. [CrossRef] 
28. Aberle, H.; Haghighi, A.P.; Fetter, R.D.; McCabe, B.D.; Magalhães, T.R.; Goodman, C.S. wishful thinking encodes a BMP type II receptor that regulates synaptic growth in Drosophila. Neuron 2002, 33, 545-558. [CrossRef]

29. Asha, H.; Nagy, I.; Kovacs, G.; Stetson, D.; Ando, I.; Dearolf, C.R. Analysis of Ras-induced overproliferation in Drosophila hemocytes. Genetics 2003, 163, 203-215.

30. Pilling, A.D.; Horiuchi, D.; Lively, C.M.; Saxton, W.M. Kinesin-1 and dynein are the primary motors for fast transport of mitochondria in Drosophila motor axons. Mol. Biol. Cell 2006, 17, 2057-2068. [CrossRef]

31. Rusten, T.E.; Vaccari, T.; Lindmo, K.; Rodahl, L.M.W.; Nezis, I.P.; Sem-Jacobsen, C.; Wendler, F.; Vincent, J.-P.; Brech, A.; Bilder, D.; et al. ESCRTs and Fab1 regulate distinct steps of autophagy. Curr. Biol. 2007, 17, 1817-1825. [CrossRef] [PubMed]

32. O'Sullivan, N.C.; Dräger, N.; O'Kane, C.J. Characterization of the Drosophila Atlastin interactome reveals VCP as a functionally related interactor. J. Genet. Genomics 2013, 6, 297-306. [CrossRef] [PubMed]

33. Stewart, B.A.; Atwood, H.L.; Renger, J.J.; Wang, J.; Wu, C.F. Improved stability of Drosophila larval neuromuscular preparations in haemolymph-like physiological solutions. J. Comp. Physiol. A 1994, 175, 179-191. [CrossRef] [PubMed]

34. Byrne, D.J.; Harmon, M.J.; Simpson, J.C.; Blackstone, C.; O'Sullivan, N.C. Roles for the VCP co-factors Npl4 and Ufd1 in neuronal function in Drosophila melanogaster. J. Genet. Genomics 2017, 44, 493-501. [CrossRef]

35. Benard, G.; Karbowski, M. Mitochondrial fusion and division: Regulation and role in cell viability. Semin. Cell Dev. Biol. 2009, 20, 356-374. [CrossRef]

36. Rowland, A.A.; Voeltz, G.K. Endoplasmic reticulum-mitochondria contacts: Function of the junction. Nat. Rev. Mol. Cell Biol. 2012, 13, 607-615. [CrossRef]

37. Li, F.; Zhang, M.; Zhang, C.; Zhou, X. Nuclear autophagy degrades a geminivirus nuclear protein to restrict viral infection in solanaceous plants. New Phytol. 2020, 225, 1746-1761. [CrossRef] [PubMed]

38. Liang, J.R.; Lingeman, E.; Ahmed, S.; Corn, J.E. Atlastins remodel the endoplasmic reticulum for selective autophagy. J. Cell Biol. 2018, 217, 3354-3367. [CrossRef] [PubMed]

39. Lin, J.R.; Shen, W.L.; Yan, C.; Gao, P.J. Downregulation of dynamin-related protein 1 contributes to impaired autophagic flux and angiogenic function in senescent endothelial cells. Arterioscler. Thromb. Vasc. Biol. 2015, 35, 1413-1422. [CrossRef] [PubMed]

40. Nezis, I.P.; Shravage, B.V.; Sagona, A.P.; Johansen, T.; Baehrecke, E.H.; Stenmark, H. Autophagy as a trigger for cell death: Autophagic degradation of inhibitor of apoptosis dBruce controls DNA fragmentation during late oogenesis in Drosophila. Autophagy 2010, 6, 1214-1215. [CrossRef]

41. Mauvezin, C.; Ayala, C.; Braden, C.R.; Kim, J.; Neufeld, T.P. Assays to monitor autophagy in Drosophila. Methods 2014, 68, 134-139. [CrossRef] [PubMed]

42. Oz-Levi, D.; Ben-Zeev, B.; Ruzzo, E.K.; Hitomi, Y.; Gelman, A.; Pelak, K.; Anikster, Y.; Reznik-Wolf, H.; Bar-Joseph, I.; Olender, T.; et al. Mutation in TECPR2 reveals a role for autophagy in hereditary spastic paraparesis. Am. J. Hum. Genet. 2012, 91, 1065-1072. [CrossRef] [PubMed]

43. Vantaggiato, C.; Crimella, C.; Airoldi, G.; Polishchuk, R.; Bonato, S.; Brighina, E.; Scarlato, M.; Musumeci, O.; Toscano, A.; Martinuzzi, A.; et al. Defective autophagy in spastizin mutated patients with hereditary spastic paraparesis type 15. Brain 2013, 136, 3119-3139. [CrossRef] [PubMed]

44. Varga, R.-E.; Khundadze, M.; Damme, M.; Nietzsche, S.; Hoffmann, B.; Stauber, T.; Koch, N.; Hennings, J.C.; Franzka, P.; Huebner, A.K.; et al. In vivo evidence for lysosome depletion and impaired autophagic clearance in hereditary spastic paraplegia type SPG11. PLoS Genet. 2015, 11, e1005454. [CrossRef] [PubMed]

45. Behrendt, L.; Kurth, I.; Kaether, C. A disease causing ATLASTIN 3 mutation affects multiple endoplasmic reticulum-related pathways. Cell. Mol. Life Sci. 2019, 76, 1433-1445. [CrossRef] [PubMed]

46. Hara, T.; Nakamura, K.; Matsui, M.; Yamamoto, A.; Nakahara, Y.; Suzuki-Migishima, R.; Yokoyama, M.; Mishima, K.; Saito, I.; Okano, H.; et al. Suppression of basal autophagy in neural cells causes neurodegenerative disease in mice. Nature 2006, 441, 885-889. [CrossRef] [PubMed]

47. Komatsu, M.; Waguri, S.; Chiba, T.; Murata, S.; Iwata, J.; Tanida, I.; Ueno, T.; Koike, M.; Uchiyama, Y.; Kominami, E.; et al. Loss of autophagy in the central nervous system causes neurodegeneration in mice. Nature 2006, 441, 880-884. [CrossRef] [PubMed]

48. Novarino, G.; Fenstermaker, A.G.; Zaki, M.S.; Hofree, M.; Silhavy, J.L.; Heiberg, A.D.; Abdellateef, M.; Rosti, B.; Scott, E.; Mansour, L.; et al. Exome sequencing links corticospinal motor neuron disease to common neurodegenerative disorders. Science 2014, 343, 506-511. [CrossRef] [PubMed] 
49. Gusdon, A.M.; Callio, J.; Distefano, G.; O’Doherty, R.M.; Goodpaster, B.H.; Coen, P.M.; Chu, C.T. Exercise increases mitochondrial complex I activity and DRP1 expression in the brains of aged mice. Exp. Gerontol. 2017, 90, 1-13. [CrossRef]

50. Klemm, R.W.; Norton, J.P.; Cole, R.A.; Li, C.S.; Park, S.H.; Crane, M.M.; Li, L.; Jin, D.; Boye-Doe, A.; Liu, T.Y.; et al. A conserved role for Atlastin GTPases in regulating lipid droplet size. Cell Rep. 2013, 3, 1465-1475. [CrossRef]

51. Mancini, C.; Roncaglia, P.; Brussino, A.; Stevanin, G.; Lo Buono, N.; Krmac, H.; Maltecca, F.; Gazzano, E.; Bartoletti Stella, A.; Calvaruso, M.A.; et al. Genome-wide expression profiling and functional characterization of SCA28 lymphoblastoid cell lines reveal impairment in cell growth and activation of apoptotic pathways. BMC Med. Genom. 2013, 6, 22. [CrossRef] [PubMed]

52. Rambold, A.S.; Kostelecky, B.; Elia, N.; Lippincott-Schwartz, J. Tubular network formation protects mitochondria from autophagosomal degradation during nutrient starvation. Proc. Natl. Acad. Sci. USA 2011, 108, 10190-10195. [CrossRef] [PubMed]

53. Gomes, L.C.; Di Benedetto, G.; Scorrano, L. During autophagy mitochondria elongate, are spared from degradation and sustain cell viability. Nat. Cell Biol. 2011, 13, 589-598. [CrossRef] [PubMed]

54. Martinez-Vicente, M. Neuronal mitophagy in neurodegenerative diseases. Front. Mol. Neurosci. 2017, 10, 64. [CrossRef]

55. Drummond, M.J.; Addison, O.; Brunker, L.; Hopkins, P.N.; McClain, D.A.; Lastayo, P.C.; Marcus, R.L. Downregulation of E3 ubiquitin ligases and mitophagy-related genes in skeletal muscle of physically inactive, frail older women: A cross-sectional comparison. J. Gerontol. Ser. A Biol. Sci. Med. Sci. 2014, 69, 1040-1048. [CrossRef] [PubMed]

56. Sun, N.; Yun, J.; Liu, J.; Malide, D.; Liu, C.; Rovira, I.I.; Holmström, K.M.; Fergusson, M.M.; Yoo, Y.H.; Combs, C.A.; et al. Measuring in vivo mitophagy. Mol. Cell 2015, 60, 685-696. [CrossRef] [PubMed]

57. Rana, A.; Oliveira, M.P.; Khamoui, A.V.; Aparicio, R.; Rera, M.; Rossiter, H.B.; Walker, D.W. Promoting Drp1-mediated mitochondrial fission in midlife prolongs healthy lifespan of Drosophila melanogaster. Nat. Commun. 2017, 8, 448. [CrossRef]

58. Kageyama, Y.; Zhang, Z.; Roda, R.; Fukaya, M.; Wakabayashi, J.; Wakabayashi, N.; Kensler, T.W.; Hemachandra Reddy, P.; Iijima, M.; Sesaki, H. Mitochondrial division ensures the survival of postmitotic neurons by suppressing oxidative damage. J. Cell Biol. 2012, 197, 535-551. [CrossRef]

59. Zhang, Q.; Hu, C.; Huang, J.; Liu, W.; Lai, W.; Leng, F.; Tang, Q.; Liu, Y.; Wang, Q.; Zhou, M.; et al. ROCK1 induces dopaminergic nerve cell apoptosis via the activation of Drp1-mediated aberrant mitochondrial fission in Parkinson's disease. Exp. Mol. Med. 2019, 51, 1-13. [CrossRef]

60. Farmer, B.C.; Walsh, A.E.; Kluemper, J.C.; Johnson, L.A. Lipid droplets in neurodegenerative disorders. Front. Neurosci. 2020, 14, 742. [CrossRef]

61. Rambold, A.S.; Cohen, S.; Lippincott-Schwartz, J. Fatty acid trafficking in starved cells: Regulation by lipid droplet lipolysis, autophagy, and mitochondrial fusion dynamics. Dev. Cell 2015, 32, 678-692. [CrossRef] [PubMed]

62. Larrea, D.; Pera, M.; Gonnelli, A.; Quintana-Cabrera, R.; Akman, H.O.; Guardia-Laguarta, C.; Velasco, K.R.; Area-Gomez, E.; Dal Bello, F.; De Stefani, D.; et al. MFN2 mutations in charcot-marie-Tooth disease alter mitochondria-Associated er membrane function but do not impair bioenergetics. Hum. Mol. Genet. 2019, 28, 1782-1800. [CrossRef] [PubMed]

63. Sandoval, H.; Yao, C.K.; Chen, K.; Jaiswal, M.; Donti, T.; Lin, Y.Q.; Bayat, V.; Xiong, B.; Zhang, K.; David, G.; et al. Mitochondrial fusion but not fission regulates larval growth and synaptic development through steroid hormone production. Elife 2014, 3, 1-23. [CrossRef]

64. Darios, F.; Mochel, F.; Stevanin, G. Lipids in the physiopathology of hereditary spastic paraplegias. Front. Neurosci. 2020, 14, 74. [CrossRef] [PubMed]

65. Deng, H.; Dodson, M.W.; Huang, H.; Guo, M. The Parkinson's disease genes pink1 and parkin promote mitochondrial fission and/or inhibit fusion in Drosophila. Proc. Natl. Acad. Sci. USA 2008, 105, 14503-14508. [CrossRef] [PubMed] 
66. Touvier, T.; De Palma, C.; Rigamonti, E.; Scagliola, A.; Incerti, E.; Mazelin, L.; Thomas, J.L.; D’Antonio, M.; Politi, L.; Schaeffer, L.; et al. Muscle-specific Drp1 overexpression impairs skeletal muscle growth via translational attenuation. Cell Death Dis. 2015, 6, e1663. [CrossRef] [PubMed]

67. Denton, K.; Mou, Y.; Xu, C.-C.; Shah, D.; Chang, J.; Blackstone, C.; Li, X.-J. Impaired mitochondrial dynamics underlie axonal defects in hereditary spastic paraplegias. Hum. Mol. Genet. 2018, 27, 2517-2530. [CrossRef] [PubMed]

(C) 2020 by the authors. Licensee MDPI, Basel, Switzerland. This article is an open access article distributed under the terms and conditions of the Creative Commons Attribution (CC BY) license (http://creativecommons.org/licenses/by/4.0/). 